\title{
Measurement of droplet impedance in segmented flow
}

\author{
Brian P. Cahill, Stefan Wiedemeier, Gunter Gastrock \\ Institut für Bioprozess- \& Analysenmesstechnik, Rosenhof, 37308 Heilbad Heiligenstadt \\ brian.cahill@iba-heiligenstadt.de
}

\begin{abstract}
:
We describe a microfluidic system for the inline contactless measurement of the impedance of droplets in segmented flow. Droplet-based microfluidics has emerged as a widespread microfluidic tool in the last ten years and has found application in chemical and biological reactors. Electrical sensing of droplet conductivity and capacitance allows the analysis of the processes that take place within the droplet. This may be the sensing of discrete objects within the droplet or the sensing of the concentration of a particular quantity in the droplet. We model the impedance of such insulated electrodes and use the model to optimize the electrode system. A system based on a polymer based microfluidic system is in advanced development.
\end{abstract}

Key words: Microfluidics, impedance spectroscopy, contactless conductivity measurement,

\section{Introduction}

For almost 15 years, contactless conductivity measurement of conductivity has been applied to measuring electrophoresis traces [1-2]. In electrophoresis, the variation of the ionic concentration is measured as a function of time without the absolute value of the conductivity being of such great importance. In recent years a couple of research groups have started using these techniques for measuring the content of droplets in segmented flow. Cahill et al. [3] presented the measurement of conductivity in segmented flow and Lederer et al. [4] described the measurement of droplet impedance in an electrowetting chip. These implementations of impedance differ from the vast majority of the applications of impedance spectroscopy, where the electrodes make galvanic contact to the liquid that is to be measured because the electrodes are insulated from the liquid to be measured. Direct contact between electrodes and the fluid medium minimizes the electrode impedance and greatly improves measurement sensitivity. The electrode impedance is adversely affected by the capacitance of insulation layers. In droplet-based microfluidic system, the droplet must be transported across the surface of the electrode without breaking up that is aided by continuous and hydrophobic surfaces. To achieve this aim, Cahill et al. [3] chose to use electrodes attached to the outer surface of thin-walled glass capillaries; the surface of glass could be readily silanized with hydrophobic alkysilanes. The sensor measured the impedance spectrum over a range of frequencies and not just a single measurement at a fixed frequency as is common in electrophoresis. This facilitated the measurement of the conductivity over a wide range. As the thickness of the capillary walls is much lower than that in capillary electrophoresis, the measurement could be performed at lower excitation amplitudes. This complexity and cost of the electronics of any dedicated electronic circuits for performing such measurements is reduced.

\section{Measurement Principle}

The general equivalent circuit describing this electrode system is shown in Fig. 1. The system consists of a tube with two insulated electrodes. $C_{w}$ is the capacitance of the insulating walls. $C_{p}$ is the parallel capacitance resulting from parasitic capacitance between the electrodes and associated cabling and from the capacitance associated with the measurement system. The quantity that is to be measured is the impedance of the fluid in the capillary that is described by the medium resistance $R_{m}$ and medium capacitance $C_{m}$.

In order to describe the behavior of the system so that we can construct a robust sensor, we will model the measurement response for the following sample values describing the equivalent circuit in Fig. $1 R_{m}=1 \mathrm{M} \Omega, C_{w}=10$ $\mathrm{pF}, C_{p}=0.070 \mathrm{pF}$ and $C_{m}=0.070 \mathrm{pF}$. The total 
impedance of the equivalent circuit in Fig. $4 \mathrm{~b}$ is given by:

$$
Z=\frac{2+j \omega R_{m}\left(C_{w}+2 C_{m}\right)}{j \omega\left(C_{w}+2 C_{p}\right)-\omega^{2} R_{m}\left(C_{m} C_{w}+C_{p} C_{w}+2 C_{p} C_{m}\right)}
$$

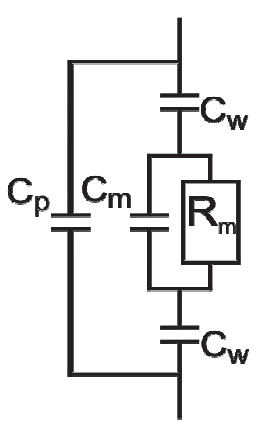

Fig. 1. Equivalent circuit diagram of the sensor where $\mathrm{Cw}$ is the capacitance of the capillary wall, $\mathrm{Cm}$ is the capacitance of the medium in the capillary, $\mathrm{Cp}$ is the parasitic capacitance and $\mathrm{Rm}$ is the resistance of the medium in the capillary.

The general form of the curves in Fig. 2 consists of three distinct frequency regions for both impedance modulus and phase angle: the low-frequency range, the mid-frequency measurement range and the high-frequency range. For the impedance modulus the lowfrequency and high-frequency ranges are decreasing curves indicative of the decrease in the impedance of a capacitor with increasing frequency. In the mid-frequency measurement range, impedance modulus value is relatively flat indicative of the measurement of the frequency-independent impedance of a resistor. The impedance phase angle behavior shows the same general trend, in the first and third sections it is $-90^{\circ}$ indicative of a pure capacitance and in the mid-frequency range it approaches a maximum value.

Fig. 2a compares the impedance of the equivalent circuit for medium resistances $R_{m}$ of $0.1,1$ and $100 \mathrm{M} \Omega$ respectively. In the midfrequency range, the impedance modulus has a value close to that of $R_{m}$, this means in the midfrequency range the resistance of the fluid in the capillary is the dominant element of the overall impedance. The mid-frequency range shifts to higher frequencies as medium conductivity increase (lower medium impedance) but without a widening of the bandwidth relative to the centre frequency. Outside of the mid-frequency measurement range there is little or no difference between the three sample curves.

Fig. $2 b$ compares the impedance of the equivalent circuit with the parallel capacitance $C_{p}$. It is clear that the mid-frequency measurement region is much narrower for higher values of $C_{p}$ and that as $C_{p}$ decreases the mid-frequency range increases in width towards higher frequencies and the maximum phase angle increases. This shows the importance of minimizing $C_{p}$ by implementing a grounded guard electrode between the measurement electrodes, by shielding cables and by optimizing the measurement electronics. Unshielded electrodes restrict the midfrequency measurement region to a relatively small bandwidth. Decreasing the value of $C_{p}$ is only effective if it is greater than $\mathrm{C}_{\mathrm{m}}$. For values of $C_{p}$ less than $C_{m}$, the increase in measurement bandwidth is relatively small. The design of the sensor and the dielectric constant of fluid determine the value of $C_{m}$.
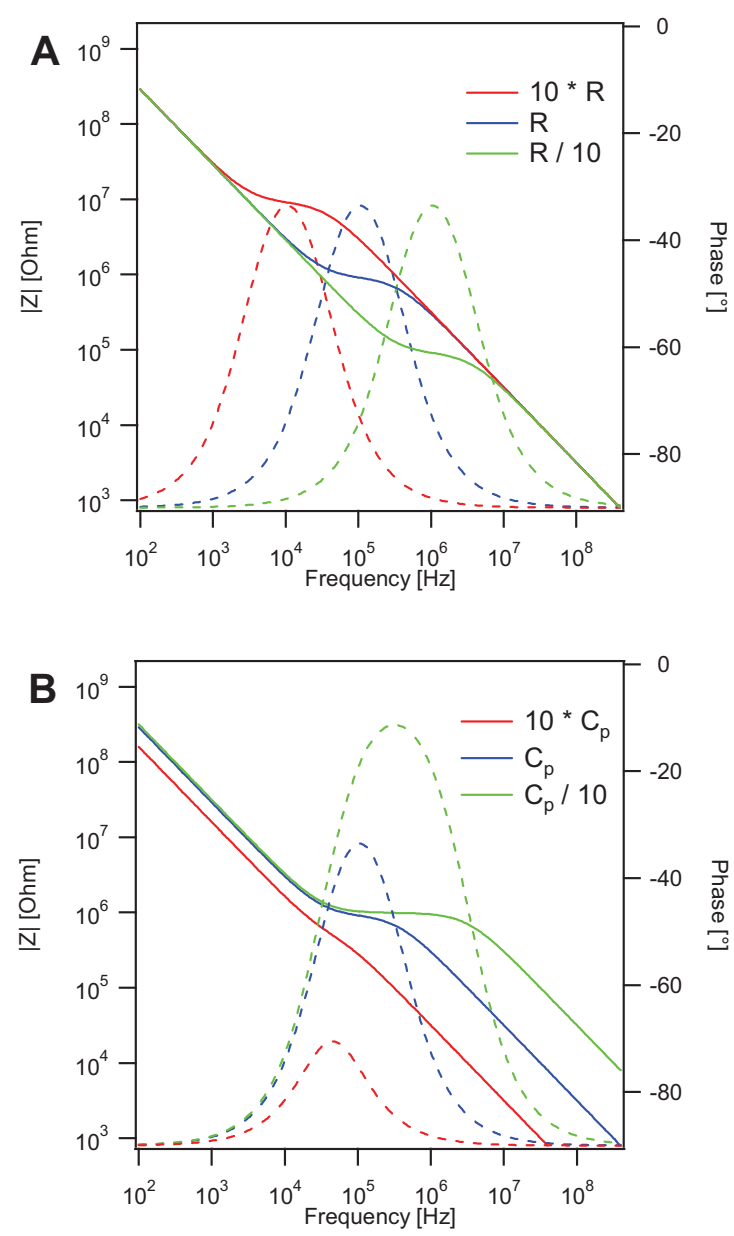

Fig. 2. (a) The dependence of the impedance modulus and impedance phase angle on medium conductivity. (b) The dependence of the impedance modulus and impedance phase angle on the parallel capacitance. 


\section{Chip Module}

The planned microfluidic system is based on a mountable and demountable microfluidic system [5] that has been designed at our institute for segmented-flow microfluidic systems.
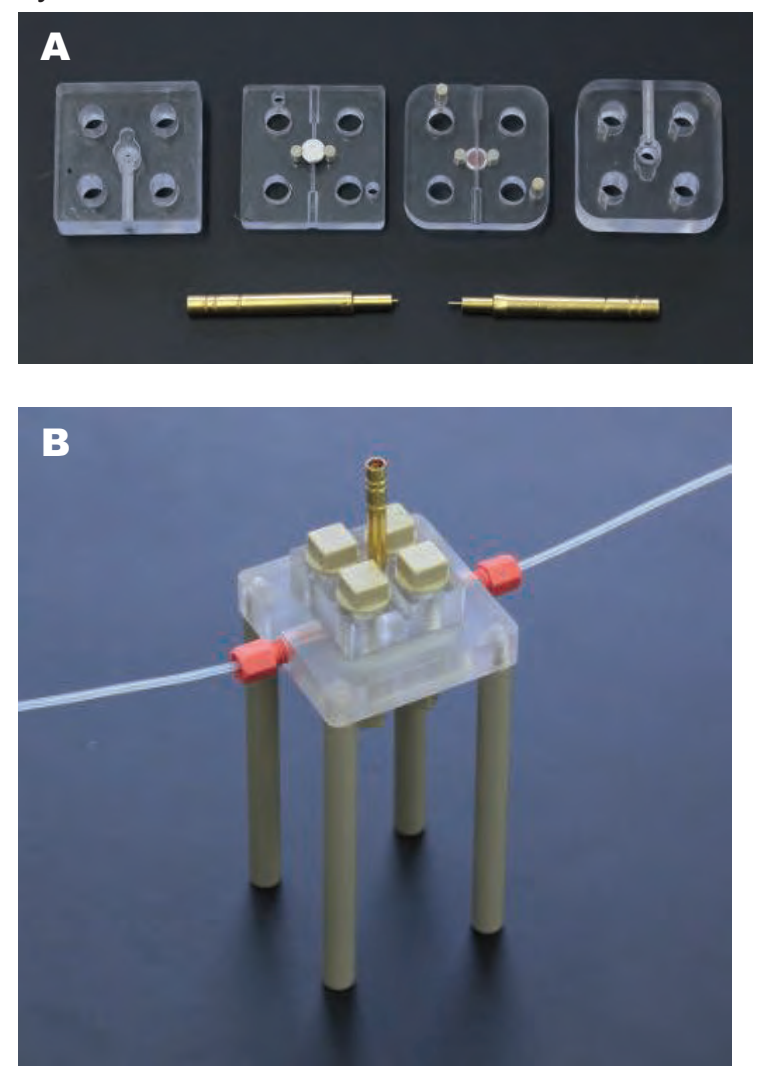

Fig. 3. (a) Sensor Prototype: Polymer chips und electrodes. (b) Assembled Sensor.

The measurement module consists of several polymer chip elements. A microfluidic channel is formed by pressing together two elements, each of which was milled to consist of a semicircular fluid channel were (Fig. 3a). The elements are machined so that the rod electrodes can be inserted to form part of the circular channel surface. Thus, the electrode geometry optimally adapts to the geometry of the fluid channel. The surfaces of the chip and the electrodes are functionalized by means of plasma process with a hydrophobic layer. This layer facilitates the integrity of droplet flow through the device and also guarantees the fluidic sealing of the mountable and demountable module. Figure $3 b$ shows the assembled measurement module.

\section{Impedance Measurement}

Measurement of solutions with various salt concentrations allows the performance of the electrode structure to be characterized. Subsequently, the measurement of the impedance response of the aqueous segments in two-phase flow serving as bioreactors allows the variation of the conductivity of the extracellular medium in the droplet to be measured. Measurements are to be firstly performed at RF frequencies with an impedance analyzer and subsequently with a lower cost measurement setup that is better suited to high-speed measurement of droplets.

\section{References}

[1] A. J. Zemann, E. Schnell, D. Volgger and G. K. Bonn, "Contactless Conductivity Detection for Capillary Electrophoresis," Anal. Chem., vol. 70, pp. 563-567, 1998.

[2] P. Kuban and P. C. Hauser, "Capacitively coupled contactless conductivity detection for microseparation techniques - recent developments," Electrophoresis, vol. 32, pp. 3042, 2011.

[3] B. P. Cahill, R. Land, T. Nacke, M. Min, and D. Beckmann, Contactless sensing of the conductivity of aqueous droplets in segmented flow, Sensors \& Actuators B, vol. 159, pp. 286293, 2011.

[4] T. Lederer, S. Clara, B. Jakoby and W. Hilber, "Integration of impedance spectroscopy sensors in a digital microfluidic platform", Microsyst. Technol., vol. 18, pp. 1163-1180, 2012.

[5] G. Gastrock, A. Grodrian, K. Lemke, S. Wiedemeier, and R. Römer (2010) Montier- und demontierbares Mikrofluidiksystem und Verfahren zur Flutung des Systems, European Patent application: EP 2248588 A1. 